\title{
A Novel One-pot Method for the Preparation of \\ Pyrazoles by 1,3-Dipolar Cycloadditions of Diazo \\ Compounds Generated In Situ
}

\author{
Varinder K. Aggarwal, ${ }^{\dagger}$ Javier de Vicente ${ }^{\dagger}$ and Roger V. Bonnert \\ School of Chemistry, Bristol University, Cantock's Close, Bristol BS8 1TS, UK \\ v.aggarwal@bristol.ac.uk
}

RECEIVED DATE (to be automatically inserted after your manuscript is accepted if required according to the journal that you are submitting your paper to)

$†$ School of Chemistry, Bristol University, Cantock’s Close, Bristol BS8 1TS, UK.

† AstraZeneca R\&D Charnwood, Medicinal Chemistry, Bakewell Road, Loughborough, Leics LE11 5RH, UK.

\section{CONTENTS}

\section{General Methods}

2. Synthesis of 3,5-Disubstituted Pyrazoles

3. Preparation of 3-Substituted Pyrazoles

4. NMR Spectras

5. References 


\section{General Methods}

Flash chromatography was performed on silica gel (Merck Kiesegel $60 \mathrm{~F}_{254} 230-400$ mesh). TLC was carried out on aluminium backed silica plates $\left(0.2 \mathrm{~mm}, 60 \mathrm{~F}_{254}\right)$ which were developed using standard visualising agents: UV fluorescence (254 and $366 \mathrm{~nm}$ ), ninhidrin/ $\Delta$, phosphomolybdic acid/ $\Delta$, anisaldehyde $/ \Delta$, permanganate/ $\Delta$. Melting points (m.p.) were determined on a Khöfler hot stage. Infrared spectras were recorded on a Perkin-Elmer Spectrum One FT-IR spectrometer using an ATR sample accessory unless otherwise specified. Only selected absorbencies $\left(\boldsymbol{\bullet}_{\max } / \mathrm{cm}^{-1}\right)$ are reported. Optical rotations were measured using a Perkin-Elmer $241 \mathrm{MC}$ polarimeter. $[\alpha]_{D}^{20}$ values are given in $10^{-1} \mathrm{deg} \mathrm{mL} \mathrm{mg}^{-1} .{ }^{1} \mathrm{H}$ NMR spectras were recorded at 270, 300 or $400 \mathrm{MHz}$ on Delta GX/270, Eclipse300 or Delta GX/400 instruments at rt unless otherwise specified. Chemical shifts $\left(\delta_{\mathrm{H}}\right)$ are quoted in parts per million (ppm) and referenced to TMS. ${ }^{13} \mathrm{C}$ NMR spectras were recorded at 68,75 or $100 \mathrm{MHz}$ on Delta GX/270, Eclipse-300 or Delta GX/400 instruments at rt unless otherwise specified. Chemical shifts $\left(\delta_{\mathrm{C}}\right)$ are quoted in parts per million ( $\left.\mathrm{ppm}\right)$, referenced to the appropriate residual solvent peak and are assigned as s, d, t, q for $\mathrm{C}, \mathrm{CH}, \mathrm{CH}_{2}$ and $\mathrm{CH}_{3}$. Degenerate peaks were prefixed by the number of carbons. DEPT ${ }^{13} \mathrm{C}$ NMR spectras are reported instead of simple ${ }^{13} \mathrm{C}$ NMR spectras in those cases when quaternary carbons were not recorded in ${ }^{13} \mathrm{C}$ NMR spectras, most probably due to longer relaxation times which is a consequence of the neighboring nitrogen atoms present in highly conjugated systems. ${ }^{1}$ Low resolution mass spectras $(\mathrm{m} / \mathrm{z})$ were recorded on a Micromass Analytical Autospec spectrometer with only molecular ions $\left(\mathrm{M}^{+}\right.$or $\left.[\mathrm{M}+\mathrm{H}]^{+}\right)$, and major peaks being reported with intensities quoted as percentages of the base peak. High-resolution mass spectras were recorded on a Micromass Analytical Autospec spectrometer. Microanalyses were performed using a Carlo Erba EA1108. GC-MS analysis were performed using an Agilent 6890 apparatus equipped with a capillary column HP-5MS (HP190915-433, 5\% Phenyl Methyl Siloxane, $30 \mathrm{~m} \times 250 \mu \mathrm{m} \times 0.25 \mu \mathrm{m}$ nominal) under the following conditions: helium $1 \mathrm{~mL} / \mathrm{min}$ (constant flow mode), injector $250^{\circ} \mathrm{C}$ (splitless 
mode), detector EI (agilent MSD 5973), oven $70^{\circ} \mathrm{C}(3 \mathrm{~min}), 15^{\circ} \mathrm{C} / \mathrm{min}(15.3 \mathrm{~min}), 300^{\circ} \mathrm{C}(8 \mathrm{~min})$. All chemicals were purchased from common chemical suppliers and used as delivered.

\section{Synthesis of 3,5-Disubstituted Pyrazoles}

\section{Representative Procedure for the Preparation of 3-Phenyl-5-substituted Pyrazoles (table 1)}

Benzaldehyde was added to a solution of $p$-toluenesulphonylhydrazide (279 mg, $1.5 \mathrm{mmol})$. After stirring for $3 \mathrm{~h}$ at $\mathrm{RT}$, a solution of $5 \mathrm{~N} \mathrm{NaOH}(300 \cdot \mathrm{L}, 1.5 \mathrm{mmol})$ was added and the mixture was stirred for a further $20 \mathrm{~min}$. Phenylacetylene $(823 \cdot \mathrm{L}, 7.5 \mathrm{mmol})$ was added and the mixture was stirred at $50^{\circ} \mathrm{C}$ for $48 \mathrm{~h}$. The volatiles were evaporated under reduced pressure and the residue was dissolved in a 1:1 mixture of water-ethyl acetate $(70 \mathrm{~mL})$. The organic layer was separated and dried over $\mathrm{MgSO}_{4}$. After filtration and removal of the solvent under reduced pressure, the crude material was purified by flash chromatography (eluent petroleum ether/ethyl acetate 3:1) to afford 292 (202 mg, $61 \%)$ as an off white solid.

\section{3,5-Diphenyl-1H-pyrazole (table 1, entry 1) ${ }^{2}$}

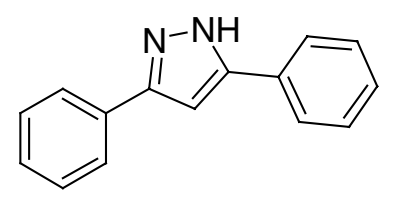

White solid (61\% yield); eluent petroleum ether/ethyl acetate 3:1, $\mathrm{R}_{f}=0.35$; m.p. 201-203•C (petroleum ether/ethyl acetate) (lit., $\left.{ }^{2} 201 \bullet \mathrm{C}\right) ;{ }^{1} \mathrm{H} \mathrm{NMR}\left(270 \mathrm{MHz}, \mathrm{CDCl}_{3}\right) \bullet 6.85(1 \mathrm{H}, \mathrm{s}, \mathrm{Pz} \mathrm{H}-4), 7.28-$ $7.42(6 \mathrm{H}, \mathrm{m}, \mathrm{Ph}), 7.74(4 \mathrm{H}, \mathrm{dd}, J=6.6$ and $1.6 \mathrm{~Hz}, \mathrm{Ph}) ;{ }^{13} \mathrm{C} \mathrm{NMR}\left(68 \mathrm{MHz}, \mathrm{CDCl}_{3}\right) \cdot 100.1(\mathrm{~d}), 125.7$ (4d), 128.2 (2d), 128.9 (4d), 134.4 (2s), 148.7; IR • ${ }_{\max } / \mathrm{cm}^{-1} 2829,1460,1272,1180,1074,974,974$, 750, 685; MS m/z (EI) $220\left(\mathrm{M}^{+}, 100\right), 191$ (28), 165 (8), 104 (8), 77 (13).

\section{4-(5-Phenyl-1H-3-pyrazolyl)benzonitrile (table 1, entry 2)}<smiles>N#Cc1ccc(-c2cc(-c3ccccc3)[nH]n2)cc1</smiles> 
Yellow needles (61\% yield); eluent DCM/MeOH 9:1, $\mathrm{R}_{f}=0.45 ;$ m.p. $250-252 \cdot \mathrm{C}$ (methanol); ${ }^{1} \mathrm{H}$ NMR $\left(300 \mathrm{MHz}, 105 \cdot \mathrm{C}, d_{6}\right.$-DMSO) • $7.18(1 \mathrm{H}, \mathrm{s}, \mathrm{Pz} \mathrm{H}-4), 7.34\left(1 \mathrm{H}, \mathrm{t}, J=7.3 \mathrm{~Hz}, \mathrm{H}_{\mathrm{p}}-\mathrm{Ph}\right), 7.45(2 \mathrm{H}, \mathrm{t}$, $\left.J=7.3 \mathrm{~Hz}, \mathrm{H}_{\mathrm{m}}-\mathrm{Ph}\right), 7.80(4 \mathrm{H}, \mathrm{m}), 8.01\left(2 \mathrm{H}, \mathrm{d}, J=8.3 \mathrm{~Hz}, \mathrm{H}_{\mathrm{o}}-\mathrm{ArCN}\right), 11.98(1 \mathrm{H}, \mathrm{br} . \mathrm{s}, \mathrm{N} H) ;{ }^{13} \mathrm{C} \mathrm{NMR}$ (DEPT, $75 \mathrm{MHz}, 105 \bullet \mathrm{C}, d_{6}$-DMSO) • $101.4(\mathrm{~d}), 125.9$ (2d), $126.4(2 \mathrm{~d}), 128.6$ (d), 129.3 (2d), 133.1 (2d); IR $\bullet_{\max } / \mathrm{cm}^{-1} 2862,2223,1608,1456,1268,1179,1058,974,838,755,678 ; \mathrm{MS} \mathrm{m} / \mathrm{z}(\mathrm{EI}) 245\left(\mathrm{M}^{+}\right.$, 100), 218 (38), 190 (12), 123 (12), 89 (10), 77 (16), 63 (6); HRMS: found 245.0955, $\mathrm{C}_{16} \mathrm{H}_{11} \mathrm{~N}_{3}$ requires 245.0953. Anal. Calc'd for $\mathrm{C}_{16} \mathrm{H}_{11} \mathrm{~N}_{3}$ : C, 78.35; H, 4.52; N, 17.13. Found: C, 78.20; H, 4.18; N, 17.10.

\section{3-(4-Methoxyphenyl)-5-phenyl-1H-pyrazole (table 1 , entry 3$)^{3}$}

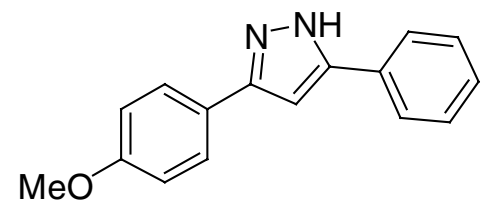

Colourless cubic solid (51\% yield); eluent petroleum ether/ethyl acetate 3:1, $\mathbf{R}_{f}=0.20$; m.p. 160162•C (methanol) (lit., $\left.{ }^{3} 166-168 \bullet C\right) ;{ }^{1} \mathrm{H} \mathrm{NMR}\left(400 \mathrm{MHz}, \mathrm{CDCl}_{3}\right) \cdot 3.84\left(3 \mathrm{H}, \mathrm{s}, \mathrm{CH}_{3}\right), 6.76(1 \mathrm{H}, \mathrm{s}, \mathrm{Pz}$ $\mathrm{H}-4), 6.94\left(2 \mathrm{H}, \mathrm{d}, J=8.8 \mathrm{~Hz}, \mathrm{ArOCH}_{3}\right), 7.34(1 \mathrm{H}, \mathrm{tt}, J=7.1$ and $2.9 \mathrm{~Hz}, p-\mathrm{Ph}), 7.42(1 \mathrm{H}, \mathrm{tt}, J=7.5$ and $2.8 \mathrm{~Hz}, m-\mathrm{Ph}), 7.66\left(2 \mathrm{H}, \mathrm{d}, J=8.8 \mathrm{~Hz}, \mathrm{ArOCH}_{3}\right), 7.73(2 \mathrm{H}, \mathrm{dt}, J=7.5$ and $2.8 \mathrm{~Hz}, o-\mathrm{Ph}) ;{ }^{13} \mathrm{C}$ NMR (100 MHz, $\left.\mathrm{CDCl}_{3}\right) \cdot 55.4$ (q), 99.5 (d), 114.3 (2d), 123.8 (s), 125.6 (2d), 126.9 (2d), 128.0 (d), 128.7 (2d), 131.4 (s), 148.3 (s), 148.9 (s), 159.7 (s); IR • ${ }_{\max } / \mathrm{cm}^{-1} 2833,1613,1508,1458,1251,1174$, 1028, 974, 831, 760, 688; GC-MS retention time 18.14 min., m/z (EI) $250\left(\mathrm{M}^{+}, 100\right), 235$ (43), 207 (32), $178(50), 152(21), 77(21)$.

\section{3-(5-Phenyl-1H-3-pyrazolyl)pyridine (table 1, entry 4)}

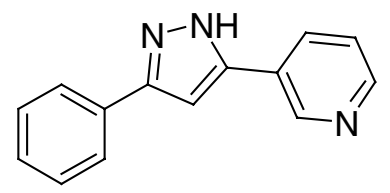

White solid (33\% yield); eluent petroleum ether/ethyl acetate $1: 3, \mathrm{R}_{f}=0.25$; m.p. 187-189•C (methanol) (lit., ${ }^{4} 187-188 \cdot \mathrm{C}$ ethanol); ${ }^{1} \mathrm{H} \mathrm{NMR}\left(270 \mathrm{MHz}, \mathrm{CDCl}_{3}\right) \cdot 6.76(1 \mathrm{H}, \mathrm{s}, \mathrm{Pz} \mathrm{H}-4), 7.18-7.34$ $(4 \mathrm{H}, \mathrm{m}), 7.57\left(2 \mathrm{H}, \mathrm{dt}, J=7.0\right.$ and $\left.1.6 \mathrm{~Hz}, \mathrm{H}_{\mathrm{o}}-\mathrm{Ph}\right), 8.01(1 \mathrm{H}, \mathrm{dt}, J=8.0$ and $1.9 \mathrm{~Hz}, \mathrm{H}-4 \mathrm{Py}), 8.34(1 \mathrm{H}$, 
d, $J=3.9 \mathrm{~Hz}, \mathrm{H}-6 \mathrm{Py}), 8.82(1 \mathrm{H}, \mathrm{d}, J=1.9 \mathrm{~Hz}, \mathrm{H}-1 \mathrm{Py}) ;{ }^{13} \mathrm{C}$ NMR (DEPT, $\left.68 \mathrm{MHz}, \mathrm{CDCl}_{3}\right) \cdot 100.2$

(d), 124.0 (d), 125.5 (2d), 128.4 (d), 128.8 (2d), 133.5 (d), 146.3 (d), 148.0 (d); IR • ${ }_{\max } / \mathrm{cm}^{-1} 3114,2827$, 1572, 1435, 960, 799, 759, 681, 662; GC-MS retention time $17.09 \mathrm{~min} ., \mathrm{m} / \mathrm{z}(\mathrm{EI}) 221\left(\mathrm{M}^{+}, 100\right), 192$ (23), 207 (32), 178 (50), $152(21), 77$ (21).

\section{Representative Procedure for the Preparation of 3-(3-Pyridinyl)-5-substituted-Pyrazoles (table}

1)

Benzaldehyde was added to a solution of $p$-toluenesulphonylhydrazide (279 mg, $1.5 \mathrm{mmol})$. After stirring for $3 \mathrm{~h}$ at RT, a solution of $5 \mathrm{~N} \mathrm{NaOH}(300 \cdot \mathrm{L}, 1.5 \mathrm{mmol})$ was added and the mixture was stirred for a further $20 \mathrm{~min}$. 3-Ethynylpyridine $(773 \mathrm{mg}, 7.5 \mathrm{mmol})$ was added and the mixture was stirred at $50^{\circ} \mathrm{C}$ for $48 \mathrm{~h}$. The solvent was evaporated under reduced pressure and the residue was dissolved in a 1:1 mixture of water-ethyl acetate $(70 \mathrm{~mL})$. The organic layer was separated and dried over $\mathrm{MgSO}_{4}$. After filtration and removal of the solvent under reduced pressure, the crude material was purified by flash chromatography (eluent petroleum ether/ethyl acetate 1:3) to afford 295 (120 mg, $36 \%)$ as an off white solid.

\section{4-[5-(3-Pyridyl)-1H-3-pyrazolyl]benzonitrile (table 1, entry 6)}

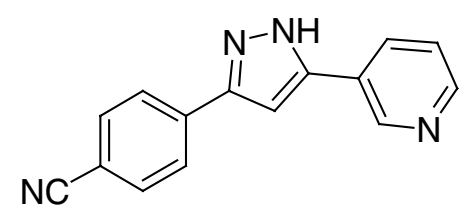

White solid (19\% yield); eluent DCM/MeOH 9:1, $\mathrm{R}_{f}=0.5 ;$ m.p. 250-252•C (DCM/MeOH); ${ }^{1} \mathrm{H}$ NMR (300 MHz, 60•C, $d_{6}$-DMSO) • $7.36(1 \mathrm{H}, \mathrm{s}, \mathrm{Pz} \mathrm{H}-4), 7.46(1 \mathrm{H}, \mathrm{dd}, J=7.9$ and $4.8 \mathrm{~Hz}, \mathrm{H}-5 \mathrm{Py}), 7.86$ $\left(2 \mathrm{H}, \mathrm{d}, J=8.2, \mathrm{H}_{\mathrm{m}}-\mathrm{ArCN}\right), 8.00\left(2 \mathrm{H}, \mathrm{d}, J=8.2 \mathrm{~Hz}, \mathrm{H}_{\mathrm{o}}-\mathrm{ArCN}\right), 8.15(1 \mathrm{H}, \mathrm{d}, J=7.9 \mathrm{~Hz}, \mathrm{H}-4 \mathrm{Py}), 8.54$ $(1 \mathrm{H}, \mathrm{dd}, J=4.8$ and $1.3 \mathrm{~Hz}, \mathrm{H}-6 \mathrm{Py}), 9.05(1 \mathrm{H}, \mathrm{d}, J=1.3 \mathrm{~Hz}, \mathrm{H}-1 \mathrm{Py}), 12.29(1 \mathrm{H}, \mathrm{br} . \mathrm{s}, \mathrm{N} H) ;{ }^{13} \mathrm{C}$ NMR $\left(\mathrm{DEPT}, 75 \mathrm{MHz}, 60 \bullet \mathrm{C}, d_{6}\right.$-DMSO) $102.1(\mathrm{~d}), 124.3$ (d), 126.5 (2d), $133.0(2 \mathrm{~d}), 133.2(\mathrm{~d}), 147.2$ (d), 149.5 (d); IR $\bullet_{\max } / \mathrm{cm}^{-1} 2853,2223,1739,1612,1570,1504,1458,1411,1380,963,840,799,705 ;$ MS m/z (EI) $246\left(\mathrm{M}^{+}, 100\right), 217$ (16), 190 (12), 164 (10); HRMS: found 246.0904, $\mathrm{C}_{15} \mathrm{H}_{10} \mathrm{~N}_{4}$ requires 246.0905. Anal. Calc'd for $\mathrm{C}_{15} \mathrm{H}_{10} \mathrm{~N}_{4}$ : C, 73.16; H, 4.09; N, 22.75. Found: C, 73.01; H, 3.70; N, 22.51. 


\section{3-[3-(4-Methoxyphenyl)-1H-5-pyrazolyl]pyridine (table 1, entry 7)}

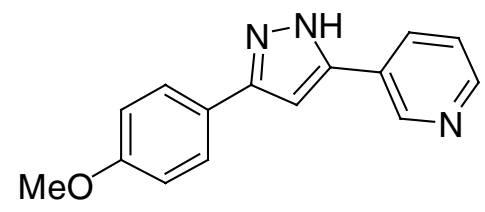

White solid (54\% yield); eluent DCM/MeOH 9:1, $\mathrm{R}_{f}=0.28 ;$ m.p. 203-205•C (methanol); ${ }^{1} \mathrm{H}$ NMR $\left(300 \mathrm{MHz}, 110 \bullet \mathrm{C}, d_{6}-\mathrm{DMSO}\right) \cdot 3.77\left(3 \mathrm{H}, \mathrm{s}, \mathrm{OCH}_{3}\right), 6.99\left(2 \mathrm{H}, \mathrm{d}, J=8.6 \mathrm{~Hz}, \mathrm{H}_{\mathrm{o}}-\mathrm{ArOCH}_{3}\right), 7.01(1 \mathrm{H}, \mathrm{s}$, Pz H-4), $7.37(1 \mathrm{H}, \mathrm{dd}, J=7.7$ and $4.8 \mathrm{~Hz}, \mathrm{H}-5 \mathrm{Py}), 7.72\left(2 \mathrm{H}, \mathrm{d}, J=8.2, \mathrm{H}_{\mathrm{m}}-\mathrm{ArOCH}_{3}\right), 8.12(1 \mathrm{H}, \mathrm{dt}, J$ = 7.9 and 1.9 Hz, H-4 Py), $8.50(1 \mathrm{H}, \mathrm{dd}, J=4.8$ and $1.3 \mathrm{~Hz}, \mathrm{H}-6 \mathrm{Py}), 9.03(1 \mathrm{H}, \mathrm{d}, J=1.3 \mathrm{~Hz}, \mathrm{H}-1$ Py), 11.74 (1H, br .s, NH); ${ }^{13} \mathrm{C}$ NMR (DEPT, $75 \mathrm{MHz}, 105 \bullet \mathrm{C}, d_{6}$-DMSO) • 55.8 (q), 99.9 (d), 115.0 (d), 124.1 (d), 127.3 (2d), 132.9 (2d), 147.1 (d), 149.0 (d); IR • ${ }_{\max } / \mathrm{cm}^{-1} 3129,3029,2835,1619,1514$, $1504,1432,1303,1256,1184,1169,1074,1025,973,839,795,697$; GC-MS retention time 18.26 min., m/z (EI) $251\left(\mathrm{M}^{+}, 100\right), 236$ (35), 208 (22), 152 (20); HRMS: found 251.1059, $\mathrm{C}_{15} \mathrm{H}_{13} \mathrm{~N}_{3} \mathrm{O}_{2}$ requires 251.1059. Anal. Calc'd for $\mathrm{C}_{15} \mathrm{H}_{13} \mathrm{~N}_{3} \mathrm{O}_{2}$ : C, 71.70; H, 5.21; N, 16.72. Found: C, 71.30; H, 5.19; $\mathrm{N}, 16.76$.

3-[5-(3-Pyridyl)-1H-3-pyrazolyl]pyridine (table 1 , entry 8) ${ }^{5}$

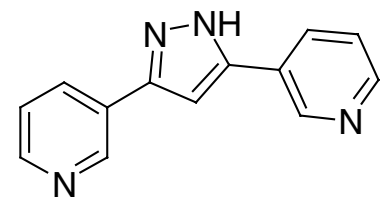

White solid (24\% yield); eluent DCM/MeOH 19:1, $\mathrm{R}_{f}=0.30$; m.p. 223-225•C (DCM/MeOH) (lit., 230.5-232•C isopropanol); ${ }^{1} \mathrm{H} \mathrm{NMR}\left(400 \mathrm{MHz}, \mathrm{CDCl}_{3}\right) \cdot 6.95(1 \mathrm{H}, \mathrm{s}, \mathrm{Pz} \mathrm{H}-4), 7.38(2 \mathrm{H}, \mathrm{dd}, J=7.7$ and 4.8 Hz, H-5 Py), 8.12 (2H, d, J = 7.7 Hz, H-4 Py), 8.46 (2H, m, H-6 Py), 8.90 (2H, m, H-1 Py); ${ }^{13} \mathrm{C}$ NMR (DEPT, $\left.100 \mathrm{MHz}, \mathrm{CDCl}_{3}\right) \cdot 100.8$ (d), 124.1 (2d), 133.6 (2d), 146.2 (2d), 148.4 (2d); IR ${ }_{\max } / \mathrm{cm}^{-1} 3113,2820,1414,1025,960,798,704,693$; GC-MS retention time $17.80 \mathrm{~min} ., \mathrm{m} / \mathrm{z}$ (EI) 222 $\left(\mathrm{M}^{+}, 100\right), 193$ (13), $166(11), 140$ (15), 118 (15), 78 (20), 63 (26), 51 (28).

\section{Preparation of 3-Substituted Pyrazoles}

Representative Procedure for the Preparation of 3-Substituted Pyrazoles (table 2) 
Benzaldehyde was added to a solution of $p$-toluenesulphonylhydrazide (279 mg, $1.5 \mathrm{mmol})$. After stirring for $3 \mathrm{~h}$ at $\mathrm{RT}$, a solution $5 \mathrm{~N} \mathrm{NaOH}(300 \cdot \mathrm{L}, 1.5 \mathrm{mmol})$ was added and the mixture was stirred for a further $20 \mathrm{~min}$. 1-Vinylimidazole $(679 \cdot \mathrm{L}, 7.5 \mathrm{mmol})$ was added and the mixture was stirred at $50^{\circ} \mathrm{C}$ for $48 \mathrm{~h}$. The volatiles were evaporated under reduced pressure and the residue was dissolved in a 1:1 mixture of water-ethyl acetate $(70 \mathrm{~mL})$. The organic layer was separated and dried over $\mathrm{MgSO}_{4}$. After filtration and removal of the solvent under reduced pressure, the crude material was purified by flash chromatography (eluent petroleum ether/ethyl acetate 3:1) to afford 3-(Phenyl)-1H-pyrazole (121 $\mathrm{mg}, 56 \%)$ as a white solid.

\section{3-(Phenyl)-1H-pyrazole (table 2 , entry 1$)^{6}$}

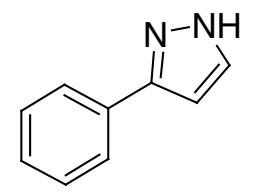

White solid (56\% yield); eluent petroleum ether/ethyl acetate $3: 1, \mathrm{R}_{f}=0.40 ;$ m.p. $102-104 \cdot \mathrm{C}$ (petroleum ether/ethyl acetate) (lit., $\left.{ }^{6} 99 \cdot \mathrm{C}\right) ;{ }^{1} \mathrm{H} \mathrm{NMR}\left(400 \mathrm{MHz}, \mathrm{CDCl}_{3}\right) \cdot 6.63(1 \mathrm{H}, \mathrm{d}, J=2.2 \mathrm{~Hz}, \mathrm{Pz}$ H-4), $7.34(1 \mathrm{H}, \mathrm{tt}, J=7.4$ and $2.6 \mathrm{~Hz}, p-\mathrm{Ph}), 7.42(1 \mathrm{H}, \mathrm{tt}, J=8.8$ and $2.0 \mathrm{~Hz}, m-\mathrm{Ph}), 7.63(1 \mathrm{H}, \mathrm{d}, J=$ $2.2 \mathrm{~Hz}, \mathrm{Pz} \mathrm{H}-5), 7.75(1 \mathrm{H}, \mathrm{d}, J=8.8 \mathrm{~Hz}, o-\mathrm{Ph}), 10.24\left(1 \mathrm{H}\right.$, br. s, NH); ${ }^{13} \mathrm{C} \mathrm{NMR}\left(68 \mathrm{MHz}, \mathrm{CDCl}_{3}\right) \bullet$ 102.7 (d), 125.9 (2d), 128.1 (d), 128.8 (2d), 132.2 (s), 133.4 (d), 149.8 (s); IR • ${ }_{\max } / \mathrm{cm}^{-1} 3160,2917$ 1455, 1353, 1071, 1047, 952, 917, 825, 749, 693; GC-MS retention time $11.35 \mathrm{~min} ., \mathrm{m} / \mathrm{z}(\mathrm{EI}) 144\left(\mathrm{M}^{+}\right.$, 100), 115 (42), 89 (22), 77 (22), 63 (14), 51 (14).

\section{3-(2-Methylphenyl)-1H-pyrazole (table 2, entry 2)}

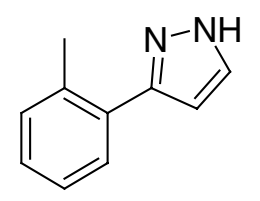

Off white solid (47\% yield); eluent petroleum ether/ethyl acetate $3: 1, \mathrm{R}_{f}=0.2$; m.p. $46-48 \cdot \mathrm{C}$ (petroleum ether/ethyl acetate); ${ }^{1} \mathrm{H} \mathrm{NMR}\left(270 \mathrm{MHz}, \mathrm{CDCl}_{3}\right) \cdot 2.41\left(3 \mathrm{H}, \mathrm{s}, \mathrm{CH}_{3}\right), 6.39(1 \mathrm{H}, \mathrm{d}, J=2.3$ Hz, Pz H-4), 7.16-7.29 (3H, m, Ar), 7.46 (1H, d, J= 7.2 Hz, o-Ar), 7.49 (1H, d, J = 2.3 Hz, Pz H-5), $11.23(1 \mathrm{H}$, br. s, $\mathrm{NH}) ;{ }^{13} \mathrm{C}$ NMR $\left(68 \mathrm{MHz} \mathrm{CDCl}_{3}\right) \cdot 20.9$ (q), 105.5 (d), 126.0 (d), 128.3 (d), 129.3 (d), 
130.9 (d), 131.6 (s), 134.2 (d), 136.2 (s), 147.2 (s); IR • $\bullet_{\max } / \mathrm{cm}^{-1}$ 3163, 2921, 1449, 1038, 953, 932, 752, 722; GC-MS retention time 11.72 min., m/z (EI) 158 (M+1 100), 130 (88), 103 (15), 77 (15), 63 (13), 51 (12); HRMS: found 158.0841, $\mathrm{C}_{10} \mathrm{H}_{10} \mathrm{~N}_{2}$ requires 158.0844. Anal. Calc'd for $\mathrm{C}_{10} \mathrm{H}_{10} \mathrm{~N}_{2}: \mathrm{C}, 75.92 ; \mathrm{H}$, 6.37; N, 17.71. Found: C, 75.99; H, 6.25; N, 18.08.

\section{3-(4-Chlorophenyl)-1H-pyrazole (table 2, entry 5) ${ }^{6}$}<smiles>Clc1ccc(-c2cc[nH]n2)cc1</smiles>

White solid (71\% yield); eluent petroleum ether/ethyl acetate $3: 1, \mathrm{R}_{f}=0.2 ;$ m.p. $184-186{ }^{\circ} \mathrm{C}$ (petroleum ether/ethyl acetate) (lit., $\left.{ }^{7} 195-197 \cdot \mathrm{C}\right) ;{ }^{1} \mathrm{H}$ NMR $\left(270 \mathrm{MHz}, \mathrm{CDCl}_{3}\right) \cdot 6.55(1 \mathrm{H}, \mathrm{d}, J=2.0$ Hz, Pz H-4), $7.31(2 \mathrm{H}, \mathrm{d}, J=8.8 \mathrm{~Hz}, o-\mathrm{Ar}), 7.55(1 \mathrm{H}, \mathrm{d}, J=2.0 \mathrm{~Hz}, \mathrm{Pz} \mathrm{H}-5), 7.64(2 \mathrm{H}, \mathrm{d}, J=8.8 \mathrm{~Hz}$, $m$-Ar), 11.81 (1H, br. s, $\mathrm{NH}) ;{ }^{13} \mathrm{C}$ NMR (68 MHz, $\left.\mathrm{CDCl}_{3}\right) \bullet 102.8$ (d), 127.2 (2d), 129.0 (2d), 130.9 (d), 132.7 (s), 133.9 (s), 148.8 (s); IR $\bullet_{\text {max }} / \mathrm{cm}^{-1}$ 3162, 2919, 1510, 1447, 1090, 1047, 1012, 953, 832, 757, 734; GC-MS retention time 13.05 min., m/z (EI) $178\left(\mathrm{M}^{+}, 100\right), 151$ (13), 115 (34), 89 (17), 75 (15), $63(13)$.

\section{4-(1H-3-Pyrazolyl)benzonitrile (table 2, entry 4)}<smiles>N#Cc1ccc(-c2cc[nH]n2)cc1</smiles>

White solid (79\% yield); eluent petroleum ether/ethyl acetate $2: 1, \mathrm{R}_{f}=0.25$; m.p. $141-143 \cdot \mathrm{C}$ (methanol); ${ }^{1} \mathrm{H}$ NMR $\left(400 \mathrm{MHz}, \mathrm{CDCl}_{3}\right) \bullet 6.71(1 \mathrm{H}, \mathrm{d}, J=2.3 \mathrm{~Hz}, \mathrm{Pz} \mathrm{H}-4), 7.65(1 \mathrm{H}, \mathrm{d}, J=2.3 \mathrm{~Hz}, \mathrm{Pz}$ H-5), $7.68(2 \mathrm{H}, \mathrm{d}, J=8.6 \mathrm{~Hz}, o-\mathrm{Ar}), 7.90(2 \mathrm{H}, \mathrm{d}, J=8.6 \mathrm{~Hz}, m-\mathrm{Ar}), 8.90(1 \mathrm{H}, \mathrm{br} . \mathrm{s}, \mathrm{N} H)$; ${ }^{13} \mathrm{C} \mathrm{NMR}(68$ $\left.\mathrm{MHz}, \mathrm{CDCl}_{3}\right) \cdot 103.6$ (d), $111.3(\mathrm{~s}), 119.0$ (s), 126.2 (2d), 131.3 (d), 132.7 (2d), 137.3 (s), 149.2 (s); IR $\cdot_{\max } / \mathrm{cm}^{-1} 3281,2222,1608,1174,1044,947,843,774$; GC-MS retention time 14.26 min., m/z (EI) 169 $\left(\mathrm{M}^{+}, 100\right), 140$ (19), 115 (14), 89 (10), 75 (11), 63 (10), 51 (8); HRMS: found 169.0644, $\mathrm{C}_{10} \mathrm{H}_{7} \mathrm{~N}_{3}$ requires 169.0639 . 


\section{3-(4-Methoxyphenyl)-1H-pyrazole (table 2 , entry 3$)^{8}$}

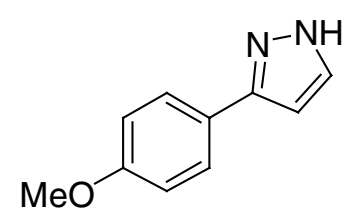

White solid (62\% yield); eluent petroleum ether/ethyl acetate $2: 1, \mathrm{R}_{f}=0.40 ;$ m.p. $128-130 \bullet \mathrm{C}$ (methanol) (lit., $\left.{ }^{8} 129-131 \cdot \mathrm{C}\right) ;{ }^{1} \mathrm{H} \mathrm{NMR}\left(270 \mathrm{MHz}, \mathrm{CDCl}_{3}\right) \cdot 3.82\left(3 \mathrm{H}, \mathrm{s}, \mathrm{OCH}_{3}\right), 6.52(1 \mathrm{H}, \mathrm{d}, J=1.9$ Hz, Pz H-4), 6.92 (2H, d, $J=8.8 \mathrm{~Hz}, o-\mathrm{Ph}), 7.58(1 \mathrm{H}, \mathrm{d}, J=1.9 \mathrm{~Hz}, \mathrm{Pz} \mathrm{H}-5), 7.66(2 \mathrm{H}, \mathrm{d}, J=8.8 \mathrm{~Hz}$, $m$-Ph), 9.40 (1H, br .s, $\mathrm{NH}) ;{ }^{13} \mathrm{C} \mathrm{NMR}\left(68 \mathrm{MHz}, \mathrm{CDCl}_{3}\right) \cdot 55.4$ (q), 102.1 (d), 114.3 (2d), 124.8 (s), 127.2 (2d), $133.7(\mathrm{~d}), 148.5$ (s), 159.6 (s); IR $\bullet_{\max } / \mathrm{cm}^{-1} 3113,2838,1610,1508,1453,1438,1248$, 1180, 1096, 1026, 831, 772; GC-MS retention time $13.41 \mathrm{~min} ., \mathrm{m} / \mathrm{z}$ (EI) $174\left(\mathrm{M}^{+}, 100\right), 159$ (64), 131 (61), 102 (12), 77 (26), 63 (10), 51 (11).

\section{3-(1H-3-Pyrazolyl)pyridine (table 2, entry 8) ${ }^{9}$}

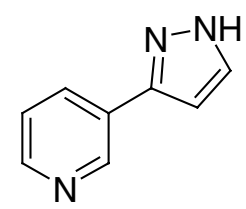

White gum (32\% yield); eluent ethyl acetate, $\mathrm{R}_{f}=0.28 ;{ }^{1} \mathrm{H}$ NMR $\left(270 \mathrm{MHz}, \mathrm{CDCl}_{3}\right) \bullet 6.68(1 \mathrm{H}, \mathrm{d}, J$ $=1.2 \mathrm{~Hz}, \mathrm{Pz} \mathrm{H}-4), 7.33$ (1H, dd, $J=7.6$ and $5.3 \mathrm{~Hz}, \mathrm{Py} \mathrm{H}-5), 7.64(1 \mathrm{H}, \mathrm{d}, J=1.2 \mathrm{~Hz}, \mathrm{Pz} \mathrm{H}-5), 8.09$ $(1 \mathrm{H}, \mathrm{d}, J=7.6 \mathrm{~Hz}, \mathrm{Py} \mathrm{H}-4), 8.56(1 \mathrm{H}, \mathrm{d}, J=5.3 \mathrm{~Hz}, \mathrm{Py} \mathrm{H}-6), 9.06(1 \mathrm{H}, \mathrm{s}, \mathrm{Py} \mathrm{H}-2) ;{ }^{13} \mathrm{C} \mathrm{NMR}(68 \mathrm{MHz}$, $\left.\mathrm{CDCl}_{3}\right) \bullet 102.9$ (d), 123.8 (d), 129.0 (s), 131.8 (d), 133.3 (d), 147.1 (d), 147.5 (s), 148.7 (d); IR $\bullet{ }_{\max } / \mathrm{cm}^{-1}$ 3138, 2907, 1418, 1028, 924, 758, 703; GC-MS retention time $12.38 \mathrm{~min} ., \mathrm{m} / \mathrm{z}(\mathrm{EI}) 145\left(\mathrm{M}^{+}, 100\right)$, 118 (10), 91 (8), 63 (15).

\section{3-(3-Furyl)-1H-pyrazole (table 2, entry 6)}

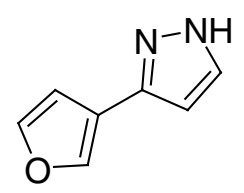

Orange solid (33\% yield); eluent petroleum ether/ethyl acetate $2: 1, \mathrm{R}_{f}=0.25$; m.p. 93-95•C (petroleum ether/ethyl acetate); ${ }^{1} \mathrm{H} \mathrm{NMR}\left(400 \mathrm{MHz}, \mathrm{CDCl}_{3}\right) \cdot 6.40(1 \mathrm{H}, \mathrm{d}, J=2.2 \mathrm{~Hz}, \mathrm{Pz} \mathrm{H}-4), 6.70$ 
$(1 \mathrm{H}, \mathrm{m}$, Fur H-4), $7.45(1 \mathrm{H}, \mathrm{t}, J=1.6 \mathrm{~Hz}$, Fur H-5), 7.57 (1H, d, J = 2.2 Hz, Pz H-5), $7.76(1 \mathrm{H}, \mathrm{m}$, Fur

H-2), 10.41 (1H, br .s, NH); ${ }^{13} \mathrm{C}$ NMR (100 MHz, $\left.\mathrm{CDCl}_{3}\right) \cdot 102.9$ (d), 109.2 (d), 118.6 (s), 132.7 (d), 139.3 (d), 142.2 (s), 143.6 (d); IR $\bullet_{\max } / \mathrm{cm}^{-1} 3139,1614,1520,1348,1192,1153,1047,985,895,872$, 796, 757; GC-MS retention time 9.80 min., m/z (EI) $134\left(\mathrm{M}^{+}, 100\right), 105$ (34), 79 (17), 51 (31); HRMS: found 134.0482, $\mathrm{C}_{7} \mathrm{H}_{6} \mathrm{~N}_{2} \mathrm{O}$ requires 134.0480.

\section{3-(2-Thienyl)-1H-pyrazole (table 2, entry 7) ${ }^{10}$}

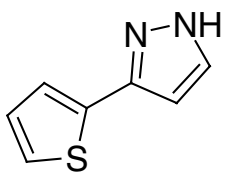

Off white solid (32\% yield); eluent petroleum ether/ethyl acetate $3: 1, \mathrm{R}_{f}=0.17$; m.p. $90-92 \bullet \mathrm{C}$ (petroleum ether/ethyl acetate) (lit., $\left.{ }^{10} 96.5-98 \cdot \mathrm{C}\right) ;{ }^{1} \mathrm{H}$ NMR $\left(270 \mathrm{MHz}, \mathrm{CDCl}_{3}\right) \cdot 6.52(1 \mathrm{H}, \mathrm{d}, J=2.3$ Hz, Pz H-4), $7.05(1 \mathrm{H}, \mathrm{dd}, J=5.0$ and $3.7 \mathrm{~Hz}, \mathrm{Th} \mathrm{H}-4), 7.25(1 \mathrm{H}, \mathrm{dd}, J=5.0$ and $1.0 \mathrm{~Hz}, \mathrm{Th} \mathrm{H}-5)$, $7.33(1 \mathrm{H}, \mathrm{dd}, J=3.7$ and $1.0 \mathrm{~Hz}$, Th $\mathrm{H}-3), 10.76(1 \mathrm{H}$, br .s, $\mathrm{NH}) ;{ }^{13} \mathrm{C} \mathrm{NMR}\left(68 \mathrm{MHz}, \mathrm{CDCl}_{3}\right) \cdot 102.7$ (d), 124.2 (d), 124.7 (d), 127.7 (d), 131.5 (d), 135.9 (s), 145.7 (s); IR • $\bullet_{\max } / \mathrm{cm}^{-1}$ 3161, 2918, 1563, 1473, 1408, 1048, 909, 845, 690; GC-MS retention time 11.59 min., m/z (EI) $150\left(\mathrm{M}^{+}, 100\right), 121$ (28), 96 (16), $78(12)$.

\section{NMR Spectras}


<smiles>c1cc(-c2ccoc2)n[nH]1</smiles>
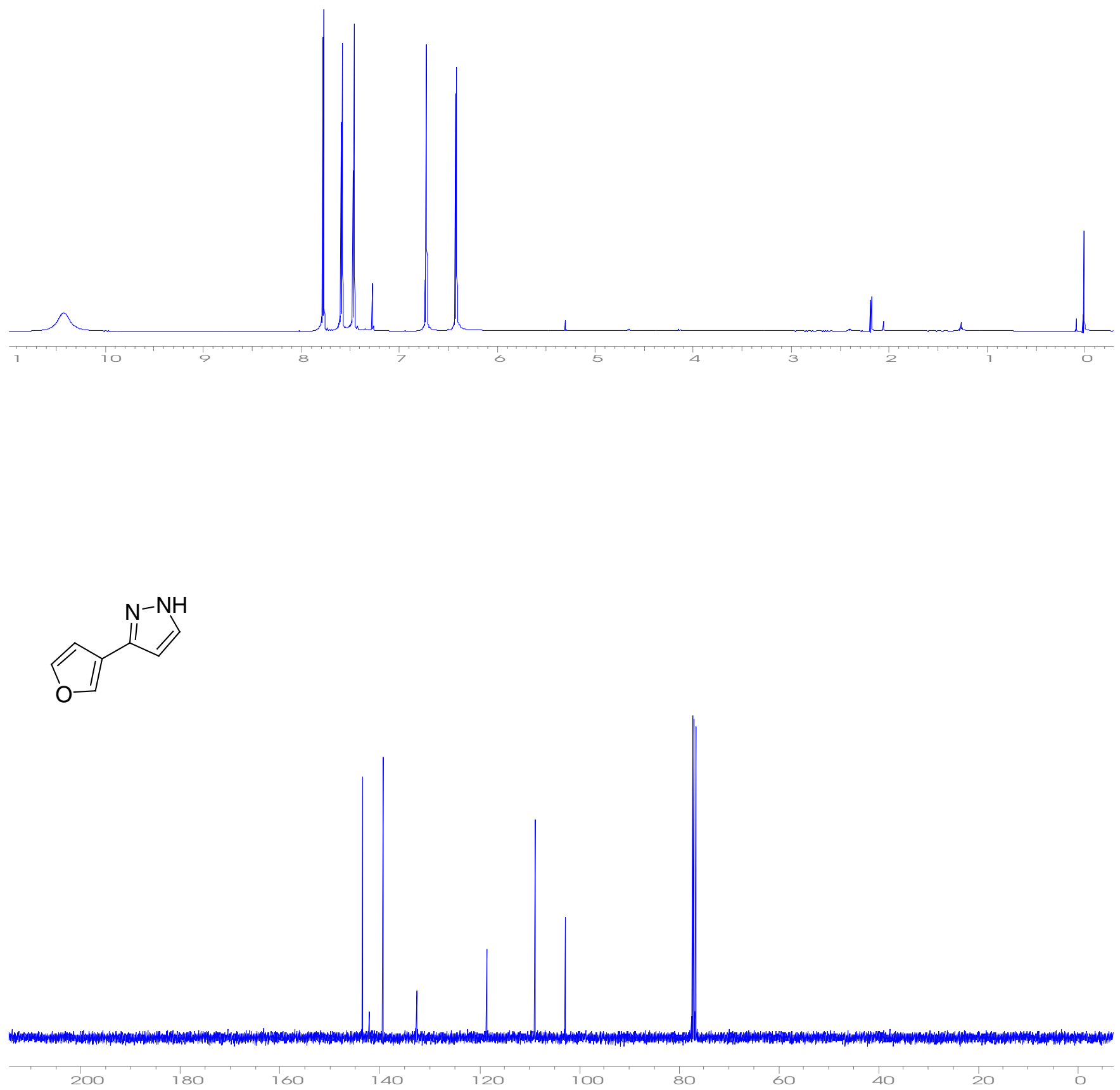

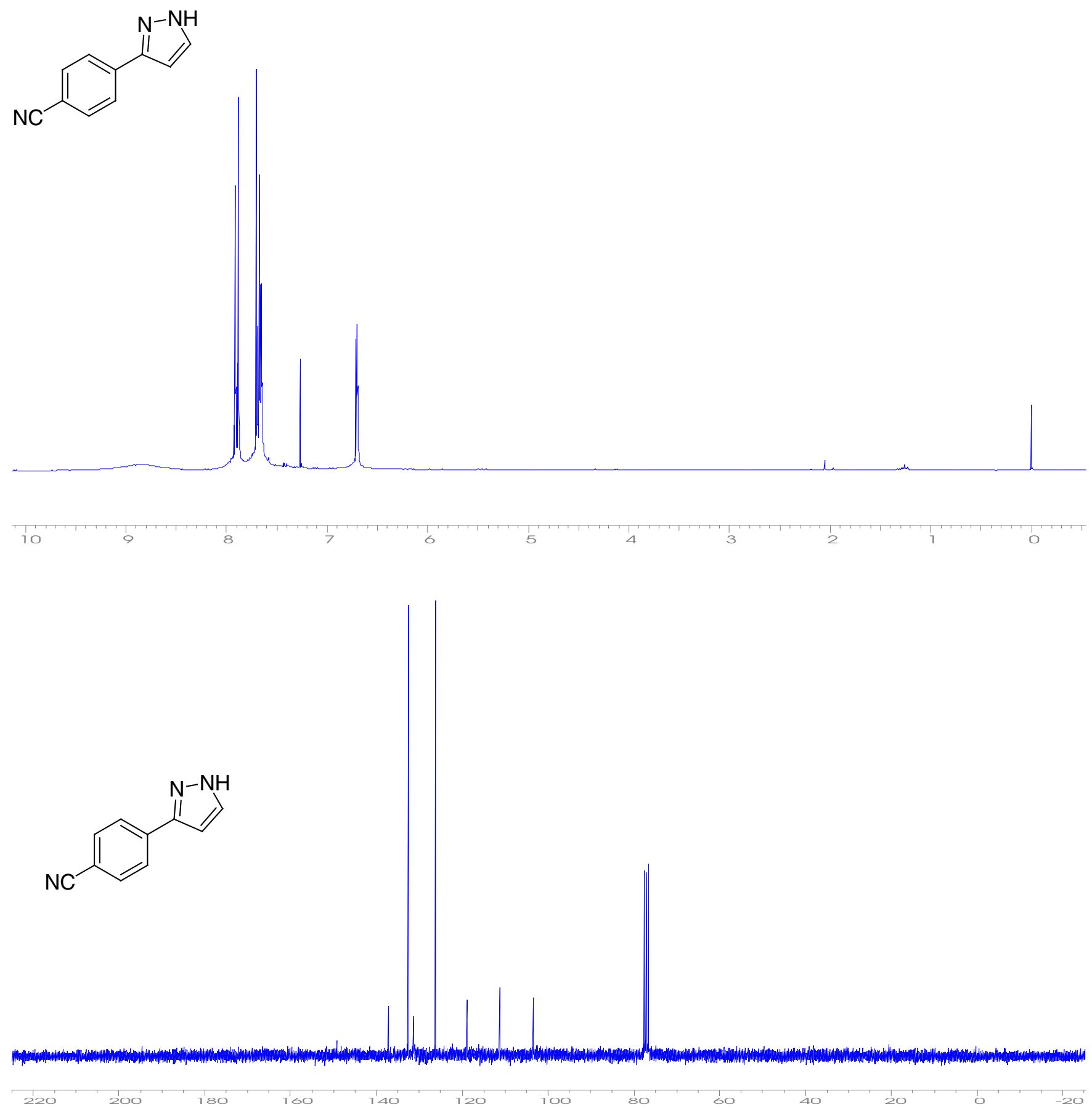

\section{References}

(1) Silverstein, R. M. Spectrometric Identification of Organic Compounds; 6th ed.; Wiley: New York, 1998. 
(2) Simon, D.; Lafont, O.; Farnoux, C. C.; Miocque, M. J. Heterocycl. Chem. 1985, 22, 1551-1557.

(3) Beam, C. F.; Foote, R. S.; Hauser, C. R. J. Chem. Soc. 1971, 1658-1660.

(4) Musante, B. Gazz. chim. ital. 1949, 79, 683-688.

(5) Ferles, M.; Liboska, R.; Trška, P. Collect. Czech. Chem. Commun. 1990, 55, 12281233.

(6) Singh, G.; IIa, H.; Junjappa, H. J. Chem. Soc., Perkin Trans. 1 1987, 1945-1950.

(7) Reddy, D. B.; Sarma, M. R.; Reddy, A. S.; Reddy, N. S. Indian J. Chem., Sect. B 2000, 39, 901-903.

(8) Grimmett, M. R.; Lim, K. H. R.; Weavers, R. T. Aust. J. Chem. 1979, 32, 2203-2213.

(9) Plate, R.; Plaum, M. J. M.; Boer, T. B.; Andrews, J. S.; Rae, D. R.; Gibson, S. Bioorg. Med. Chem. Lett. 1996, 4, 227-237.

(10) Tsuchiya, T.; Enkaku, M.; Sawanishi, H. Chem. Pharm. Bull. 1979, 27, 2188-2193. 